\title{
Radiotherapy for T3N0 glottic carcinoma without cord fixation: elective nodal irradiation or not?
}

\author{
Ryo Toya ${ }^{1}$, Ryuji Murakami², Daizo Murakami ${ }^{3}$, Tetsuo Saito', Tomohiko Matsuyama ${ }^{1}$, \\ Yutaka Toya ${ }^{3}$, Yasuyuki Yamashita ${ }^{4}$ and Natsuo Oya ${ }^{1}$ \\ ${ }^{1}$ Department of Radiation Oncology, Kumamoto University Hospital, Kumamoto, Japan \\ ${ }^{2}$ Department of Medical Imaging, Faculty of Life Sciences, Kumamoto University, Kumamoto, Japan \\ ${ }^{3}$ Department of Otolaryngology-Head and Neck Surgery, Kumamoto University Hospital, Kumamoto, Japan \\ ${ }^{4}$ Department of Diagnostic Radiology, Kumamoto University Hospital, Kumamoto, Japan \\ Correspondence to: Ryo Toya, email: ryo108@kumamoto-u.ac.jp \\ Keywords: glottic carcinoma, radiotherapy, lymph node metastasis, elective nodal irradiation, clinical target volume
}

Received: March 10, $2017 \quad$ Accepted: June 30, $2017 \quad$ Published: July 18, 2017

Copyright: Toya et al. This is an open-access article distributed under the terms of the Creative Commons Attribution License 3.0 (CC BY 3.0 ), which permits unrestricted use, distribution, and reproduction in any medium, provided the original author and source are credited.

\section{ABSTRACT}

Background: Although the T3 category has been changed in the sixth edition of the TNM staging system proposed by the Union for International Cancer Control (UICC), the appropriate clinical target volume (CTV) of elective nodal irradiation (ENI) for T3NO glottic carcinoma without cord fixation, which was formerly treated as a T1-2NO disease, is not fully discussed.

Materials and Methods: We retrospectively analyzed 64 patients staged or restaged as T3NO disease without cord fixation. All patients received irradiation to the primary lesion alone using opposed lateral fields. Surgery was performed in 10 patients without tumor regression after the delivery of $40 \mathrm{~Gy}$. The other 54 patients received a median total dose of $66 \mathrm{~Gy}$. Concurrent chemoradiotherapy (CRT) with low-dose cisplatin and UFT (low-dose CRT) and docetaxel, cisplatin, and 5-fluorouracil (TPF-CRT) were performed in 23 and 19 patients, respectively.

Results: Eighteen $(28.1 \%)$ patients suffered treatment failure; all were recorded as local failure alone. The 5-year local control rates for RT alone, low-dose CRT, and TPF-CRT groups were $51.7 \%, 61.6 \%$, and $93.8 \%$, respectively $(p=0.027)$. The 5 -year laryngeal preservation rates for RT alone, low-dose CRT, and TPF-CRT groups were $57.4 \%, 81.6 \%$, and $89.5 \%$, respectively $(p=0.048)$.

Conclusions: The rate of regional failure was zero when irradiating the primary lesion alone using opposed lateral fields. This treatment technique covers the most level III regions; hence, CTV for ENI should include level III alone.

\section{INTRODUCTION}

Glottic carcinoma is the most common laryngeal cancer [1]. Radiotherapy (RT) with or without chemotherapy is one of the recommended therapeutic approaches for the treatment of T3 disease [1,2]. After the sixth edition of the TNM staging system was proposed in 2002 by the Union for International Cancer Control (UICC), T3 glottic carcinoma included paraglottic space invasion and/or minor thyroid cartilage erosion in addition to vocal cord fixation, which was listed in the fifth edition. Subsequently, no essential revision was made, and in the newest eighth edition of the TNM staging system, T3 glottic carcinoma is defined as "tumor limited to larynx with vocal cord fixation and/or invades paraglottic space and/or inner cortex of the thyroid cartilage."

As lymph node (LN) metastasis is extremely rare, T1-2N0 glottic carcinoma has been treated with RT without elective nodal irradiation (ENI) [3]. However, ENI for levels II-IV has been traditionally recommended for T3N0 disease [4]. Although the T3 category has changed, the optimal clinical target volume (CTV) of ENI for T3N0 disease without cord fixation, which was formerly treated as a T1-2N0 disease, is not fully discussed. Before the 
introduction of the UICC sixth edition, we reported the existence of the invasion of the paraglottic space and inner cortex of the thyroid cartilage $[5,6]$. In the present study, we investigated the optimal CTV of ENI for T3N0 disease without cord fixation by evaluating the regional failure based on our hospital's database.

\section{RESULTS}

Figure 1 shows the local control (LC), laryngeal preservation (LP), and overall survival (OS) curves for patients with T3N0 glottic carcinoma without cord fixation treated using RT with or without chemotherapy over 240 months. The median follow-up duration was 69.3 months (range: 9.5-227.1 months). During the follow-up period, $46(71.9 \%)$ patients experienced tumor control and $18(28.1 \%)$ suffered treatment failure. All patients with treatment failure were recorded as local failure alone; regional and distant failure was not observed. Of the 18 patients with local failure, LP was obtained with salvage surgery in 10 patients. Overall, LP was obtained in $49(76.6 \%)$ patients. Two patients with local failure underwent neck dissection, which was confirmed as pathologically node-negative.

The 5-year LC rates for all patients were $64.9 \%$. The 5-year LC rates for RT alone; concurrent chemo-RT
(CRT) with low-dose cisplatin and UFT (low-dose CRT group); and concurrent CRT with docetaxel, cisplatin, and 5-fluorouracil (TPF-CRT group) were 51.7\%, 61.6\%, and $93.8 \%$, respectively ( $p=0.027$, Figure 1 ). The 5 -year LP rates for all patients were $74.9 \%$. The 5-year LP rates for RT alone, low-dose CRT, and TPF-CRT groups were $57.4 \%, 81.6 \%$, and $89.5 \%$, respectively $(p=0.048$, Figure 1). The 5-year OS rates for all patients were $88.4 \%$. The 5-year OS rates for RT alone, low-dose CRT, and TPF-CRT groups were $69.1 \%, 100 \%$, and $92.9 \%$, respectively ( $p=0.003$, Figure 1$)$.

All toxicities were manageable, and there were no grade 5 events. One patient experienced grade 4, six patients experienced grade 3, and eight patients experienced grade 2 myelosuppression. One patient experienced grade 3 liver toxicity. Thirteen patients experienced grade 2 gastrointestinal toxicities. Four and two patients experienced grade 2 mucositis and dermatitis, respectively.

\section{DISCUSSION}

The results of our study revealed that the rate of regional failure when irradiating primary lesions alone using opposed lateral fields for patients with T3N0 glottic carcinoma without cord fixation was zero. The
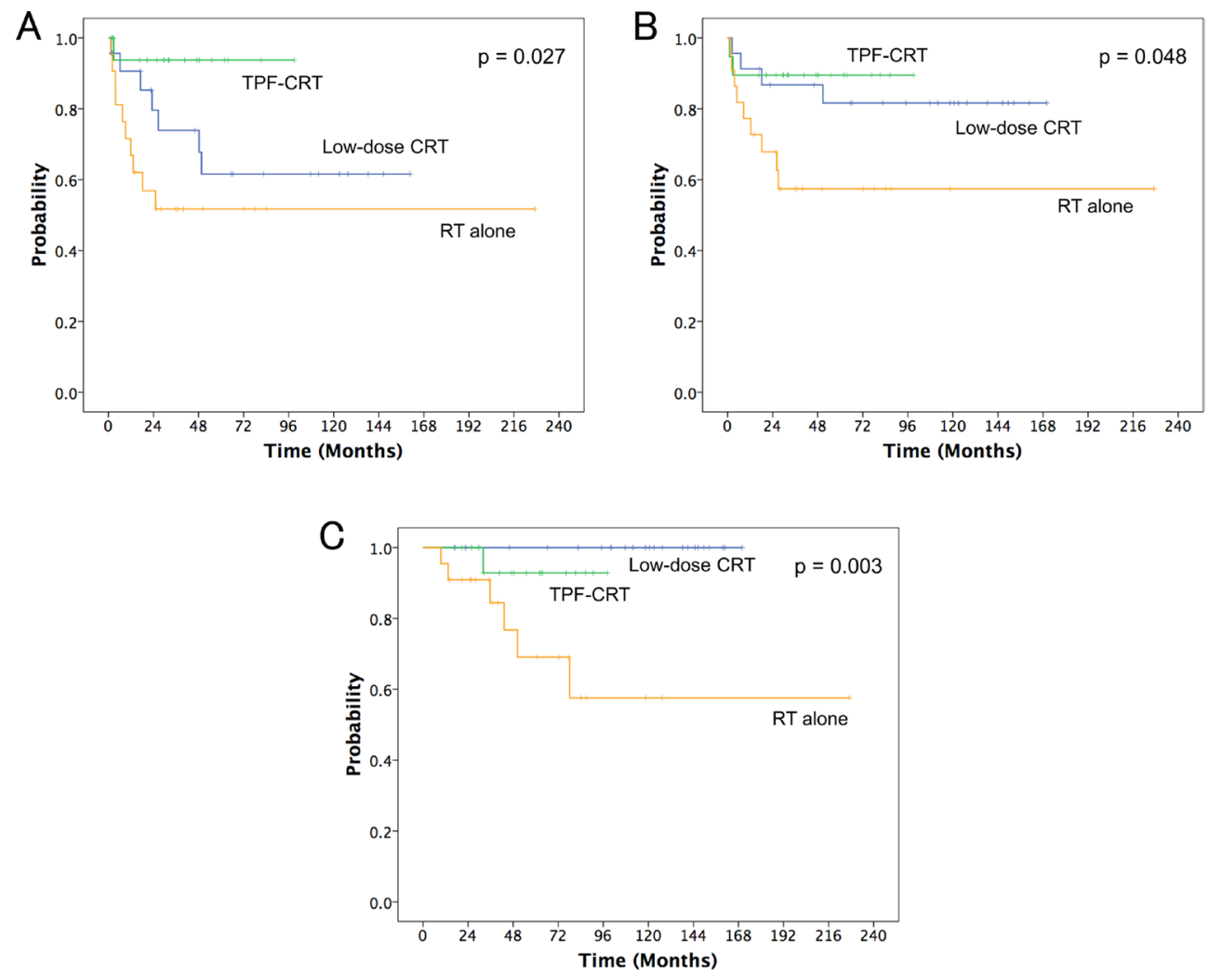

Figure 1: (A) local control, (B) laryngeal preservation, and (C) overall survival curves for the three treatment groups of radiotherapy (RT) alone, low-dose chemo-RT (CRT) with low-dose cisplatin and UFT (low-dose CRT), and concurrent CRT with docetaxel, cisplatin, and 5-fluorouracil (TPF-CRT). 
traditionally recommended CTV for ENI was based mainly on pathological examinations using palpation rather than based on imaging. In pathological analyses of the early 1990s, the incidence of LN metastasis for clinical N0 patients was reported as more than $30 \%$ [7]. However, the introduction of modern imaging techniques such as computed tomography (CT), magnetic resonance imaging (MRI), and 2-deoxy-2-[18F]fluoro-D-glucose (FDG)positron emission tomography (PET) into the nodal staging of head and neck cancer has provided excellent diagnostic accuracy $[8,9]$. Furthermore, pathological studies revealed that level III is the most commonly involved $\mathrm{cN} 0$ glottic carcinoma regions $[7,10]$. As irradiation with opposed lateral fields, which is typically used for early T1-T2N0 glottic carcinoma, covers most of the level III regions, microscopic metastasis to this level should be electively irradiated $[3,11]$. Therefore, CTV for ENI should include level III alone for T3N0 glottic carcinoma without cord fixation. Hence, irradiation to the primary lesion alone using opposed lateral fields was an appropriate treatment technique for this disease. Prior to the revision of UICC sixth edition, Pelliteri et al. [12] treated 162 T1-2N0 glottic carcinoma patients with total RT dose of 60-70 Gy using this technique. They investigated the pattern of failure and found that there was no patient with regional failure alone. Yamazaki et al. [13] performed a prospective randomized study for T1N0 glottic carcinoma using the same technique. One hundred and eighty patients were randomly allocated to either the treatment arm of total RT dose of 60-66 Gy in 2-Gy fractions or total RT dose of 56.25-63 Gy in 2.25-Gy fractions. They reported that no regional failure was observed before local failure in both treatment arms. Jones et al. [14] treated 115 patients with T1-2N0 glottic carcinoma patients with a total RT dose of 60.75-75.6 Gy using a similar technique; they irradiated the primary lesion alone with the 3-field technique delivering $90 \%$ of the total dose using opposed lateral fields with the remainder of the dose coming from an anterior field. They found no isolated regional recurrence and recommended against ENI for these diseases.

In previous reports of treatment results with RT alone for the T3 disease, the LC, LP, and OS rates were 35\%-65\%, $60 \%-75 \%$, and $45 \%-50 \%$, respectively [15-18]. In the results of the RTOG 91-11 trial, the LC, LP, and OS rates for stage III-IV laryngeal cancer were $53.6 \%$, $65.8 \%$, and $53.8 \%$ for RT alone group and $71.1 \%, 83.6 \%$, and $55.1 \%$ for concurrent CRT group, respectively [19]. Al-mamgani et al. [20] treated 170 T3 laryngeal cancer patients using RT with or without concurrent chemotherapy. The LC and OS rates were $68 \%$ and $49 \%$, respectively. Our treatment results were comparable to those of these previous reports with acceptable toxicities.

Our study has some limitations, including the retrospective design, relatively small number of patients for a long study period, and variability in RT doses and chemotherapy regimens. We could not rule out the potential influence of the surgical intervention after the delivery of $40 \mathrm{~Gy}$ on treatment outcomes of regional failure. The chemotherapy regimens were relatively uncommon in the current practice. We analyzed the pattern of failure based on patients treated with RT using opposed lateral fields. Recent planning studies suggested the dosimetric benefit of intensity-modulated RT (IMRT) for carotid artery sparing in the treatment of early glottic carcinoma [21]. Although we recommend ENI for level III alone, we cannot comment on the laterality of this level.

\section{MATERIALS AND METHODS}

\section{Patients}

This retrospective study was approved by the institutional review board of our hospital. Informed consent was obtained from all patients prior to treatment. Between November 1992 and February 2015, 515 patients with pathologically diagnosed glottic carcinoma who had undergone pretreatment physical, endoscopic, and radiological examinations were treated with $\mathrm{RT}$ with or without chemotherapy. Of these, 74 patients were staged or restaged as $\mathrm{T} 3 \mathrm{~N} 0$ without cord fixation according to the eighth edition of the UICC; 47 patients had T1-2N0 with invasion of the paraglottic space and/or inner cortex of the thyroid cartilage according to the UICC fifth edition and 27 patients had T3N0 without cord fixation according to the UICC sixth or seventh edition. Finally, 64 patients were included in this study and 10 patients were excluded from analysis; eight patients received ENI before 1999, one patient had coexisting advanced small cell lung cancer, which required simultaneous CRT, and one patient was lost to follow-up within 6 months. All patients were males and ranged in age from 43 to 88 years (median age: 69 years) at the start of their treatment.

\section{Radiological examinations}

Pretreatment examinations included ultrasonography (US), MRI, CT, and/or FDG- PET/CT. MR studies were also performed with 40 Gy irradiation for the interim assessment of the tumor regression [22].

\section{Treatment}

Between 1992 and 1998, all 9 patients were treated with RT alone. Between 1999 and 2005, we performed concurrent low-dose CRT with low-dose cisplatin and UFT in 23 patients [6]. To receive this treatment, the patients were required to be 20-80 years of age, have an Eastern Cooperative Oncology Group performance status (PS) of 0-2, a life expectancy of at least 3 months, adequate hematolotic (WBC $\geq 4000 / \mathrm{mm}^{3}, \mathrm{Hb} \geq 10 \mathrm{~g} / \mathrm{dl}$, Plts $\geq 100$ $\left.000 / \mathrm{mm}^{3}\right)$ and renal $(\mathrm{Cr} \leq 1.2 \mathrm{mg} / \mathrm{dl}, \mathrm{CCr} \geq 60 \mathrm{ml} / \mathrm{min})$ function, no severe complications, and provide informed 
consent. The patients received an intravenous infusion of cisplatin $\left(4 \mathrm{mg} / \mathrm{m}^{2}\right)$ for $1 \mathrm{~h}$ prior to delivery of the daily fraction and orally took the fixed dose of UFT (150 mg of tegafur three times a day; $450 \mathrm{mg}$ daily) after meals starting on the first day of irradiation and continuing for 4 weeks. Nine patients received RT alone in this period because they did not meet the eligibility criteria.

Between 2006 and 2015, we performed concurrent TPF-CRT with docetaxel, cisplatin, and 5-fluorouracil in 19 patients [23]. For inclusion, patients had to be 20-80 years of age, have a PS of $0-2$, a life expectancy of at least 3 months, adequate hematolotic (WBC $\geq 3500 / \mathrm{mm}^{3}$, $\mathrm{ANC} \geq 2000 / \mathrm{mm}^{3}, \mathrm{Hb} \geq 9.5 \mathrm{~g} / \mathrm{dl}$, Plts $\geq 100$ 000/. $\mathrm{mm}^{3}$ ), renal $(\mathrm{Cr} \leq 1.2 \mathrm{mg} / \mathrm{dl}, \mathrm{CCr} \geq 60 \mathrm{ml} / \mathrm{min})$, and hepatic (TB $1.5 \mathrm{mg} / \mathrm{dl}$, AST $\leq 2 \times \mathrm{ULN}$, ALT $\leq 2 \times \mathrm{ULN})$ function, no severe complications, and provide informed consent. The patients received an intravenous infusion of docetaxil $\left(50 \mathrm{mg} / \mathrm{m}^{2}\right)$ for $1 \mathrm{~h}$ on day 1 . More than $1 \mathrm{~h}$ after completing the docetaxel infusion, $5-\mathrm{FU}\left(600 \mathrm{mg} / \mathrm{m}^{2} /\right.$ day $)$ was delivered by continuous intravenous infusion on days $1-5$. Cisplatin $\left(60 \mathrm{mg} / \mathrm{m}^{2}\right)$ was given intravenously on day 4. Two cycles of chemotherapy were repeated every 4 weeks. Four patients received RT alone in this period because they did not meet the eligibility criteria.

RT was delivered using a 3-4 MV linear accelerator in daily fractions ranging from 1.8 to 2 Gy. All 64 patients received irradiation to the primary lesion alone with opposed lateral fields (Figure 2). RT field extended from the thyroid notch superiorly to the inferior border of the cricoid to $1 \mathrm{~cm}$ behind the posterior aspect of the thyroid cartilage with a 1-cm fall-off anteriorly [11, 24]. Field borders were modified for each patient depending on the anatomical extent of the particular lesion. The median field size was $36 \mathrm{~cm}^{2}$ (range: $25-49 \mathrm{~cm}^{2}$, corresponding to $5 \times 5-7 \times 7 \mathrm{~cm}$ ). After the delivery of $40 \mathrm{~Gy}$, otolaryngologists and radiation oncologists, by consensus, performed interim assessment to evaluate tumor regression from endoscopic and radiological examinations [6, 22]. In 10 patients, the tumors showed no regression and surgery was performed: total laryngectomy $(n=4)$ and laryngeal preservation surgery $(n=6)$. The remaining 52 patients received a median total dose of 66 Gy (range: 60-72 Gy);

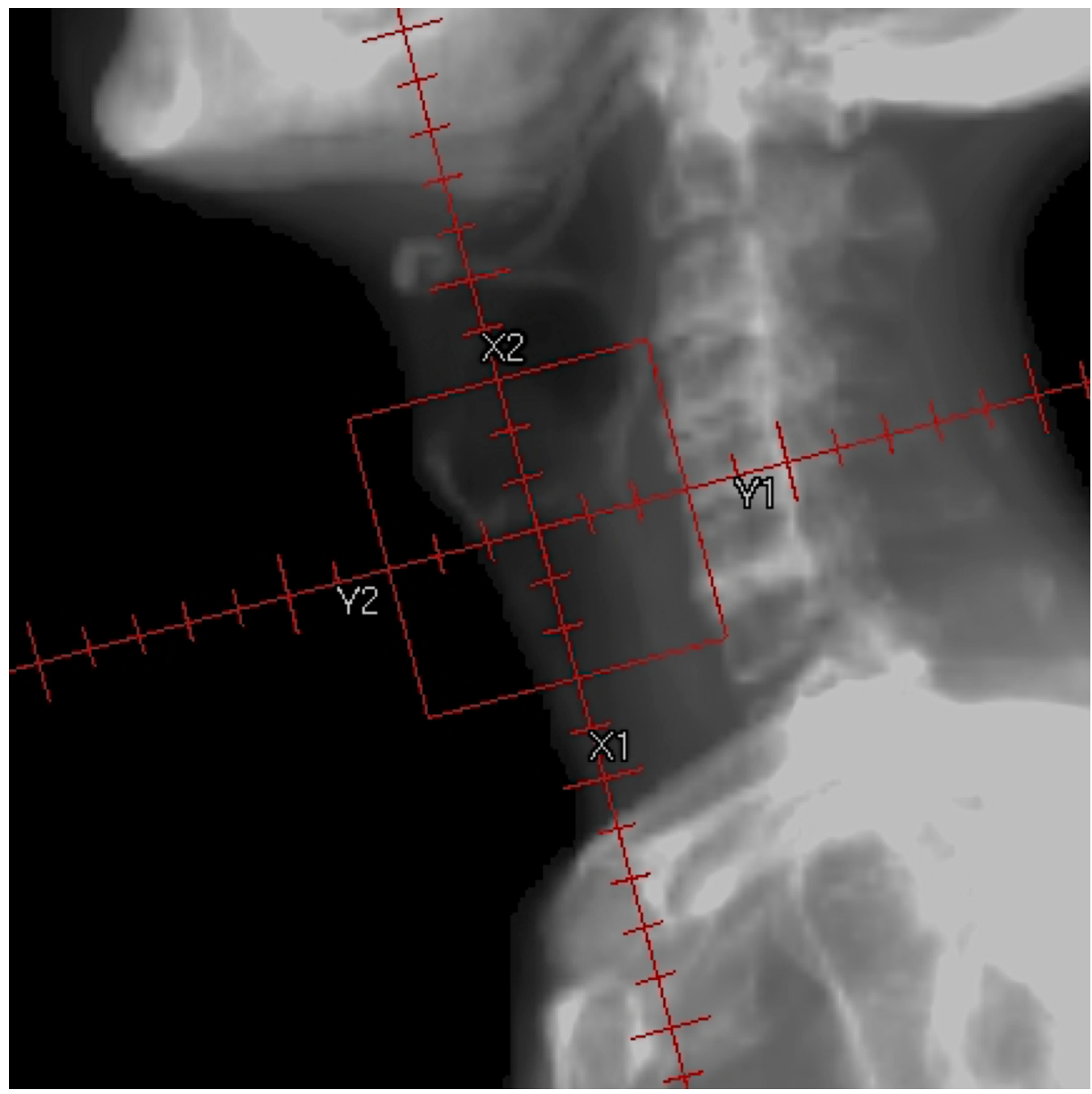

Figure 2: Radiotherapy field for the T3N0 glottic carcinoma without cord fixation. 
a median total dose of 70 Gy (range: 63.8-72 Gy) for RT alone, 68 Gy (range: 60-72 Gy) for low-dose CRT, and 66 Gy (range: 64-66 Gy) for TPF-CRT groups, respectively.

\section{Follow-up}

After the completion of treatment, patients were evaluated every 1-2 months during the first year, every 3 months during the second year, and every 6 months thereafter. All patients underwent physical and endoscopic examinations at each follow-up visit. When a followup endoscopy detected a suspicious lesion, biopsy specimens were examined to rule out tumor recurrence. Post-treatment US, MR imaging, and/or CT studies were also performed within 1-2 months after the completion of treatment and every 6 months thereafter or when clinically indicated. A diagnosis of treatment failure was based on the pathology specimen results. Failures were classified as involving the primary lesion (local), neck LN metastases (regional), or distant metastases (distant). Treatment-associated toxicities were graded according to the Common Terminology Criteria of Adverse Events Version 4.0 (CTCAE V4.0).

\section{Statistical analysis}

LC, LP, and OS rates were calculated from the initiation of treatment using the Kaplan-Meier method. Patients who underwent surgery and had a pathological complete response to the treatment were censored at the time of surgery for the calculation of LC according to Mendenhall et al. [16]. The effect of chemotherapy was evaluated using log-rank statistics. Differences with $p$-values $<0.05$ were considered statistically significant. Statistical calculations were performed using SPSS software, version 24.0 (SPSS Inc., Chicago, IL, USA).

\section{Author contributions}

RT developed the study design; collected, analyzed, and interpreted the data; performed statistical analysis; and wrote the manuscript. RM developed the study design; collected, analyzed, and interpreted the data; and revised the manuscript. DM and YT collected the data. TS, TM, YY, and NO developed the study design and interpreted the data. All authors have read and approved the final manuscript.

\section{CONFLICT OF INTEREST}

The authors declare that they have no competing interests.

\section{REFERENCES}

1. Doornaert P, Terhaard CH, Kaanders JH, Dutch National Platform Radiotherapy Head and Neck Cancer. Treatment of T3 laryngeal cancer in the Netherlands: a national survey. Radiat Oncol. 2015; 10:134.

2. National Comprehensive Cancer Network. NCCN Clinical Practice Guidelines in Oncology, Head and Neck Cancers (Version 1.2017).

3. Mendenhall WM, Amdur RJ, Morris CG, Hinerman RW. T1T2N0 squamous cell carcinoma of the glottic larynx treated with radiation therapy. J Clin Oncol. 2001; 19:4029-36.

4. Gregoire V, Coche E, Cosnard G, Hamoir M, Reychler H. Selection and delineation of lymph node target volumes in head and neck conformal radiotherapy. Proposal for standardizing terminology and procedure based on the surgical experience. Radiother Oncol. 2000; 56:135-50.

5. Murakami R, Nishimura R, Baba Y, Furusawa M, Ogata N, Yumoto E, Yamashita Y. Prognostic factors of glottic carcinomas treated with radiation therapy: value of the adjacent sign on radiological examinations in the sixth edition of the UICC TNM staging system. Int J Radiat Oncol Biol Phys. 2005; 61:471-5.

6. Murakami R, Nishimura R, Baba Y, Yumoto E, Oya N, Yamashita Y. Concurrent chemoradiation therapy with lowdose CDDP and UFT for glottic carcinomas: evaluation using the sixth edition of the UICC TNM staging system. Acta Oncol. 2006; 45:162-7.

7. Candela FC, Shah J, Jaques DP, Shah JP. Patterns of cervical node metastases from squamous carcinoma of the larynx. Arch Otolaryngol Head Neck Surg. 1990; 116:432-5.

8. Murakami R, Uozumi H, Hirai T, Nishimura R, Shiraishi S, Ota K, Murakami D, Tomiguchi S, Oya N, Katsuragawa S, Yamashita Y. Impact of FDG-PET/CT imaging on nodal staging for head-and-neck squamous cell carcinoma. Int $\mathrm{J}$ Radiat Oncol Biol Phys. 2007; 68:377-82.

9. Lyhne NM, Johansen J, Kristensen CA, Andersen E, Primdahl H, Andersen LJ, Boje CR, Jensen AR, Overgaard J. Pattern of failure in 5001 patients treated for glottic squamous cell carcinoma with curative intent - A population based study from the DAHANCA group. Radiother Oncol. 2016; 118:257-66.

10. Ma H, Lian M, Feng L, Li P, Hou L, Liu H, Chen X, Huang Z, Fang J. Management of cervical lymph nodes for cN0 advanced glottic laryngeal carcinoma and its long-term results. Acta Otolaryngol. 2014; 134:952-8.

11. Mendenhall WM, Parsons JT, Brant TA, Stringer SP, Cassisi NJ, Million RR. Is elective neck treatment indicated for T2N0 squamous cell carcinoma of the glottic larynx? Radiother Oncol. 1989; 14:199-202.

12. Pellitteri PK, Kennedy TL, Vrabec DP, Beiler D, Hellstrom M. Radiotherapy. The mainstay in the treatment of early glottic carcinoma. Arch Otolaryngol Head Neck Surg. 1991; 117:297-301.

13. Yamazaki H, Nishiyama K, Tanaka E, Koizumi M, Chatani M. Radiotherapy for early glottic carcinoma (T1N0M0): results of prospective randomized study of radiation fraction size and overall treatment time. Int $\mathrm{J}$ Radiat Oncol Biol Phys. 2006; 64:77-82. 
14. Jones DA, Mendenhall CM, Kirwan J, Morris CG, Donnan A, Holwerda S, Kraus ST, Mann CJ, Grant JR, Donnan B, Mendenhall WM. Radiation therapy for management of $\mathrm{t} 1-\mathrm{t} 2$ glottic cancer at a private practice. Am J Clin Oncol. 2010; 33:587-90.

15. Nguyen-Tan PF, Le QT, Quivey JM, Singer M, Terris DJ, Goffinet DR, Fu KK. Treatment results and prognostic factors of advanced T3-4 laryngeal carcinoma: the University of California, San Francisco (UCSF) and Stanford University Hospital (SUH) experience. Int J Radiat Oncol Biol Phys. 2001; 50:1172-80.

16. Mendenhall WM, Parsons JT, Mancuso AA, Pameijer FJ, Stringer SP, Cassisi NJ. Definitive radiotherapy for T3 squamous cell carcinoma of the glottic larynx. J Clin Oncol. 1997; 15:2394-402.

17. Parsons JT, Mendenhall WM, Mancuso AA, Cassisi NJ, Stringer SP, Million RR. Twice-a-day radiotherapy for T3 squamous cell carcinoma of the glottic larynx. Head Neck. 1989; 11:123-8.

18. Hinerman RW, Mendenhall WM, Morris CG, Amdur RJ, Werning JW, Villaret DB. T3 and T4 true vocal cord squamous carcinomas treated with external beam irradiation: a single institution's 35-year experience. Am J Clin Oncol. 2007; 30:181-5.

19. Forastiere AA, Zhang Q, Weber RS, Maor MH, Goepfert H, Pajak TF, Morrison W, Glisson B, Trotti A, Ridge JA, Thorstad W, Wagner H, Ensley JF, et al. Long-term results of RTOG 91-11: a comparison of three nonsurgical treatment strategies to preserve the larynx in patients with locally advanced larynx cancer. J Clin Oncol. 2013; 31:845-52.

20. Al-Mamgani A, Tans L, van Rooij P, Levendag PC. A single-institutional experience of 15 years of treating T3 laryngeal cancer with primary radiotherapy, with or without chemotherapy. Int J Radiat Oncol Biol Phys. 2012; 83:1000-6.

21. Ward MC, Pham YD, Kotecha R, Zakem SJ, Murray E, Greskovich JF. Clinical and dosimetric implications of intensity-modulated radiotherapy for early-stage glottic carcinoma. Med Dosim. 2016; 41:64-9.

22. Toya R, Murakami R, Saito T, Murakami D, Matsuyama T, Baba Y, Nishimura R, Hirai T, Semba A, Yumoto E, Yamashita Y, Oya N. Radiation therapy for nasopharyngeal carcinoma: the predictive value of interim survival assessment. J Radiat Res. 2016; 57:541-7.

23. Katori H, Tsukuda M. Comparison of induction chemotherapy with docetaxel, cisplatin, and 5-fluorouracil (TPF) followed by radiation vs concurrent chemoradiotherapy with TPF in patients with locally advanced squamous cell carcinoma of the head and neck. Clin Oncol (R Coll Radiol). 2005; 17:148-52.

24. Mendenhall WM, Parsons JT, Million RR, Fletcher GH. T1-T2 squamous cell carcinoma of the glottic larynx treated with radiation therapy: relationship of dose-fractionation factors to local control and complications. Int J Radiat Oncol Biol Phys. 1988; 15:1267-73. 